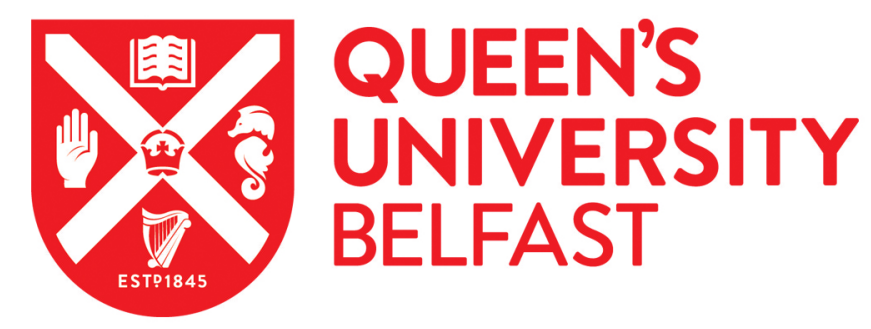

\title{
Gamma-ray Spectra and Enhancement Factors for Positron Annihilation with Core Electrons
}

Green, D. G., \& Gribakin, G. F. (2015). Gamma-ray Spectra and Enhancement Factors for Positron Annihilation with Core Electrons. Physical Review Letters, 114(9), [093201]. https://doi.org/10.1103/PhysRevLett.114.093201

Published in:

Physical Review Letters

Document Version:

Peer reviewed version

Queen's University Belfast - Research Portal:

Link to publication record in Queen's University Belfast Research Portal

Publisher rights

(c) 2015 American Physical Society

\section{General rights}

Copyright for the publications made accessible via the Queen's University Belfast Research Portal is retained by the author(s) and / or other copyright owners and it is a condition of accessing these publications that users recognise and abide by the legal requirements associated with these rights.

Take down policy

The Research Portal is Queen's institutional repository that provides access to Queen's research output. Every effort has been made to ensure that content in the Research Portal does not infringe any person's rights, or applicable UK laws. If you discover content in the Research Portal that you believe breaches copyright or violates any law, please contact openaccess@qub.ac.uk. 


\title{
$\gamma$-ray spectra and enhancement factors for positron annihilation with core electrons
}

\author{
D. G. Green* and G. F. Gribakin ${ }^{\dagger}$ \\ Department of Applied Mathematics and Theoretical Physics, \\ Queen's University Belfast, Belfast, BT71NN, Northern Ireland, United Kingdom
}

(Dated: March 9, 2015)

\begin{abstract}
Many-body theory is developed to calculate the $\gamma$-spectra for positron annihilation in noblegas atoms. Inclusion of electron-positron correlation effects and core annihilation gives spectra in excellent agreement with experiment [Iwata et al., Phys. Rev. Lett. 79, 39 (1997)]. The calculated correlation enhancement factors $\gamma_{n l}$ for individual electron orbitals $n l$ are found to scale with the ionization energy $I_{n l}$ (in $\mathrm{eV}$ ), as $\gamma_{n l}=1+\sqrt{A / I_{n l}}+\left(B / I_{n l}\right)^{\beta}$, where $A \approx 40 \mathrm{eV}, B \approx 24 \mathrm{eV}$ and $\beta \approx 2.3$.

PACS numbers: 78.70.Bj, 34.80.Pa, 34.80.-i, 34.8.Uv
\end{abstract}

Introduction.-This Letter shows that many-body theory (MBT) provides accurate $\gamma$-ray spectra for positron annihilation with valence and core electrons of noble-gas atoms, and establishes firmly the fractions of core annihilation. It uncovers a simple scaling of the enhancement factors, which increase the annihilation probability beyond the independent-particle approximation (IPA), with the electron ionization energy.

Low-energy positrons annihilate predominantly on the valence electrons in atoms. Small fractions of positrons can, however, tunnel through the repulsive nuclear potential and annihilate with core electrons [1]. The two-photon annihilation $\gamma$-ray spectrum is Dopplerbroadened by the electron velocity distribution in the states involved. In particular, annihilation on tightlybound core electrons results in distinct features at larger Doppler shifts $[2,3]$. Its signal shows high elemental specificity [4], allowing to study vacancies and other defects in metals and semiconductors [5-7]. Annihilation on core electrons enables positron-induced Augerelectron spectroscopy (PAES) [8-11] and time-resolved PAES [12], to study the dynamics of catalysis, corrosion, and surface alloying [13]. Coincident measurements of the $\gamma$-rays and Auger electrons yields $\gamma$-ray spectra for individual core orbitals [14, 15].

Interpretation of experiments relies heavily on theoretical input, e.g., the relative annihilation probabilities for core electrons of various atoms in PAES [16]. However, positron annihilation in many-electron systems is characterised by strong electron-positron correlations, which affect the positron wave function and electron-positron annihilation vertex. Correlations lead to dramatic enhancements of positron annihilation rates in heavier noblegas atoms, compared with the single-particle approximation (see [17] and references therein), and influence the shapes of the $\gamma$-ray spectra [18-20]. For atomic systems correlations can be included systematically by MBT methods [17, 21]. MBT provided early insights into positron annihilation in metals by considering positrons in an electron gas $[22,23]$. These works introduced the concept of enhancement factors (EF), which mea- sure the increase of the electron density at the positron. Subsequently, density functional theories were developed for condensed-matter systems [24, 25]. They describe positron states and annihilation in real materials, often using parametrizations of the correlation energy and EF for the positron in electron gas from MBT [26]. The EF are particularly large $(\sim 10)$ for the valence electrons, but also significant for the core electrons [27]. They correct the IPA annihilation probabilities and $\gamma$-spectra $[2,16]$. However, they also lead to spurious effects in the spectra [6], and show deficiencies when benchmarked against accurate calculations [28].

Positron interaction with noble-gas atoms has been studied thoroughly in experiment by measuring the scattering cross sections and annihilation rates. This system is ideal for testing the ability of theory to account for correlations. An extensive comparison with the data attests the accuracy of our MBT approach [17]. One outstanding issue is the annihilation $\gamma$-ray spectra of $\mathrm{Ar}, \mathrm{Kr}$ and Xe [3], that have till now eluded theoretical description. In this work we extend the MBT approach to the $\gamma$-ray spectra. The calculation of the valence and core annihilation yields excellent agreement with experiment, including the large Doppler shifts where the core contribution dominates. The MBT also provides "exact" EF $\gamma_{n l}$ for individual electron orbitals $n l[29]$.

Theory.- In the dominant process, a positron annihilates with an electron in state $n$ to form two $\gamma$-ray photons of total momentum $\mathbf{P}$ [30]. In the centre-of-mass frame the two $\gamma$-rays have equal energies $m c^{2}=511 \mathrm{keV}$ (neglecting the initial positron and electron energies $\varepsilon$ and $\varepsilon_{n}$ ). In the laboratory frame the photon energies are Doppler shifted by $\epsilon \leq P c / 2$, and their spectrum is

$$
w_{n}(\epsilon)=\frac{1}{c} \int_{2|\epsilon| / c}^{\infty} \int_{\Omega_{\mathbf{P}}}\left|A_{n \varepsilon}(\mathbf{P})\right|^{2} \frac{d \Omega_{\mathbf{P}}}{(2 \pi)^{3}} P d P,
$$

where $A_{n \varepsilon}(\mathbf{P})$ is the annihilation amplitude [18]. Figure 1 shows the main contributions to this amplitude: the zeroth-order vertex (IPA), and the first- and higher-order (' $\Gamma$-block') corrections, which account for the attractive electron-positron interaction at short range (see [17-19, 
31, 32] for details). The total spectrum, which is probed in experiment, is the sum of the spectra of individual atomic orbitals $n l, w(\epsilon)=\sum_{n l} w_{n l}(\epsilon)$.

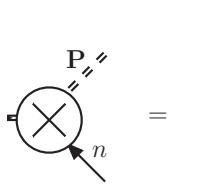

(a)

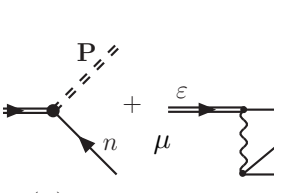

(b)

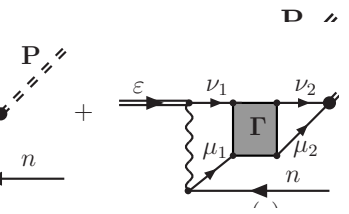

(c)
FIG. 1. Amplitude of positron annihilation with an electron in state $n$ : (a) zeroth-order, (b) first-order, and (c) ' $\Gamma$-block' corrections. Double lines labelled $\varepsilon$ represent the incident positron; single lines labelled $\nu(\mu)$ represent positron (excited electron) states, which are summed over; lines labelled $n$ represent holes in the atomic ground state; wavy lines represent the electron-positron Coulomb interaction, and doubledashed lines represent the two $\gamma$-ray photons. The $\Gamma$-block is the sum of the electron-positron ladder diagram series [17, 21].

The fully-correlated incident positron quasiparticle wave function $\psi_{\varepsilon}$ is obtained from the Dyson equation $\left(H_{0}+\hat{\Sigma}_{\varepsilon}\right) \psi_{\varepsilon}=\varepsilon \psi_{\varepsilon}$, where $H_{0}$ is the positron Hamiltonian in the field of the Hartree-Fock (HF) ground state atom, and $\hat{\Sigma}_{\varepsilon}$ is the positron self-energy operator which represents the positron-atom correlation potential. This potential accounts for polarization of the atom by the positron and for virtual positronium formation (represented by the $\Gamma$-block), both of which contribute to the positron-atom attraction (see $[17,21]$ for details).

The positron annihilation rate in a gas is parameterized by the dimensionless effective number of electrons, $Z_{\text {eff }}$ [33]. For an orbital $n l$, it is $Z_{\text {eff }, n l}=\int_{-\infty}^{\infty} w_{n l}(\epsilon) d \epsilon$. $Z_{\mathrm{eff}, n l}$ for valence orbitals is usually greater than the actual number of electrons, owing to the positron-atom attraction and vertex corrections.

The positron self-energy diagrams and the annihilation amplitude contain sums over the intermediate excited electron and positron states. We calculate them numerically using a basis set $40 \mathrm{~B}$-splines of order 6 , in a spherical box of radius 30 a.u. The maximum angular momentum of the intermediate states is $l_{\max }=15$, and we extrapolate to $l_{\max } \rightarrow \infty$ as in [18] (see [17, 31, 32] for details).

Results.-The annihilation $\gamma$-ray spectra for Ar, $\mathrm{Kr}$ and Xe were measured with room-temperature positrons in a Penning-Malmberg trap [3]. That work also showed that the IPA [Fig. 1 (a)] overestimates both the full width at half maximum (FWHM) of the spectra and the fraction of core annihilation. Ref. [18] showed that the firstorder correction [Fig. 1 (b)] narrowed the spectrum, but was insufficient to describe the experiment.

The full calculation presented in this work highlights the importance of higher-order corrections [Fig. 1 (c)], especially for the valence electrons. The MBT also shows that the self-energy that affects the positron wave func-

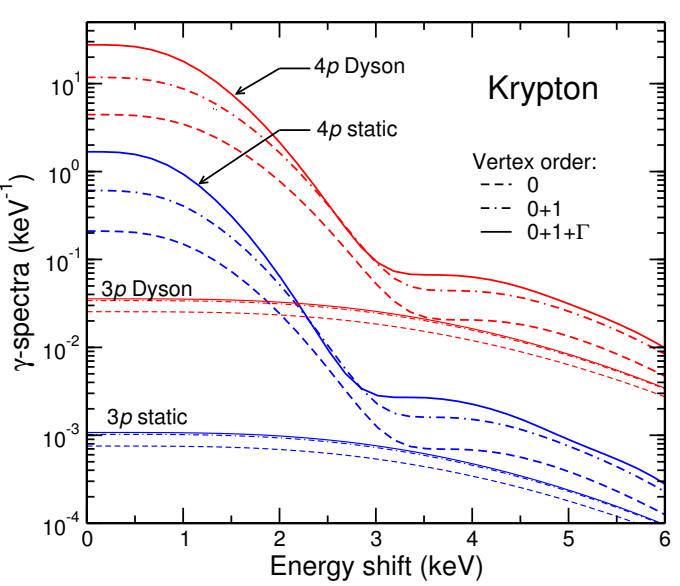

FIG. 2. Annihilation $\gamma$-ray spectra for the $4 p$ valence and $3 p$ core electron orbitals in $\mathrm{Kr}$, calculated using the positron wave function in the static field of the HF atom, and with the account of the correlation potential $\hat{\Sigma}_{\varepsilon}$ (Dyson), and with various approximations for the annihilation vertex [Fig. 1]: zeroth-order, dashed curves ("0"); zeroth- and first-order, chain curves (" $0+1$ "); full vertex, solid curves (" $0+1+\Gamma$ ").

tion (double line in Fig. 1) and the correlation corrections to the vertex [diagrams (b) and (c)] have strikingly different effects on the spectra. As an example, Fig. 2 presents the spectra for the valence $4 p$ orbital and a core $3 p$ orbital in $\mathrm{Kr}$ [34]. It shows that the vertex corrections enhance the annihilation by almost an order of magnitude for the valence electrons and by about $50 \%$ for the core orbital. The higher-order corrections [Fig. 1 (c)] are much more prominent for the valence electrons. Vertex corrections also lead to a significant narrowing of the spectrum for the valence electrons.

In contrast, improving the positron wave function (i.e., using the Dyson orbital instead of the static HF state) uniformly increases the annihilation signal. This increase is due to the build-up of the positron density in the vicinity of the atom caused by the positron-atom attraction. The magnitude of this effect is similar for the valence and core electrons. However, unlike the vertex corrections, it is sensitive to the atomic environment and the positron energy (e.g., the low-energy annihilation in $\mathrm{Ar}, \mathrm{Kr}$ and $\mathrm{Xe}$ is strongly enhanced by the positron virtual states $[17])$.

Figure 3 shows the spectra for positron annihilation on individual subshells of $\mathrm{Ar}, \mathrm{Kr}$ and $\mathrm{Xe}$, calculated with the full amplitude (Fig. 1) using the Dyson $s$-wave positron state of thermal momentum $k=0.04$ a.u. The narrowly peaked valence spectra dominate the total spectra at low Doppler shifts. The tightly-bound and faster moving core electrons produce broader $\gamma$-ray spectra. Note also that most individual spectra include multiple 'shoulders'. They are caused by the oscillations of the electron or- 


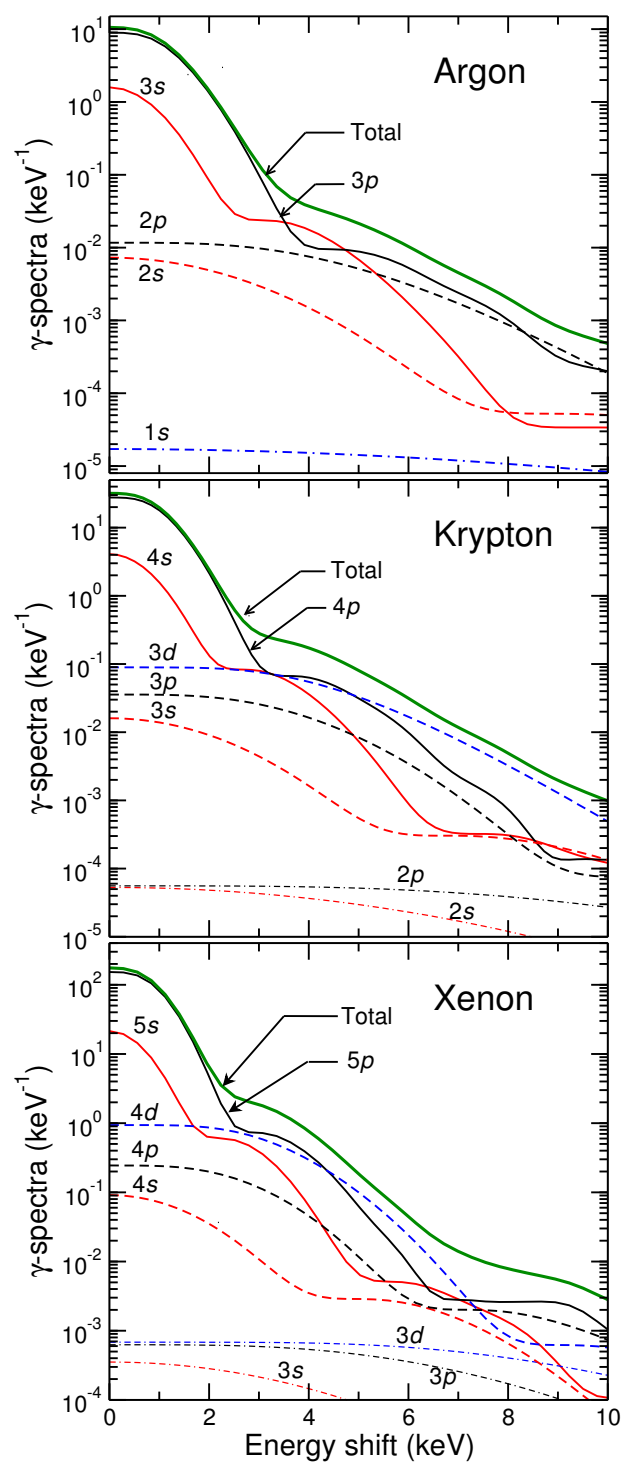

FIG. 3. Calculated $\gamma$-spectra for positron annihilation on individual subshells $n l$ in $\mathrm{Ar}, \mathrm{Kr}$ and Xe: valence $n s, n p$, (solid black and red lines); core $(n-1) s,(n-1) p$, and $(n-1) d$ (dashed lines); inner core $(n-2) s,(n-2) p$, and $(n-2) d$ (dash-dash-dotted lines); and total spectra (thick solid green line). All spectra are obtained using the full annihilation vertex (Fig. 1) and Dyson positron wave function.

bitals due to their orthogonality to the lower-lying states. In this way the spectra of the valence orbitals contain high-momentum components characteristic of the core orbitals. Overall, the total $\gamma$-spectra retain the characteristics of both the valence and core contributions.

Figure 4 shows the calculated total spectra convolved with the detector resolution function and normalized to the experimental data at zero Doppler shifts [3]. For each atom the valence component underestimates the experimental spectrum at higher energies. Inclusion of the core brings the theoretical spectra into close agreement with

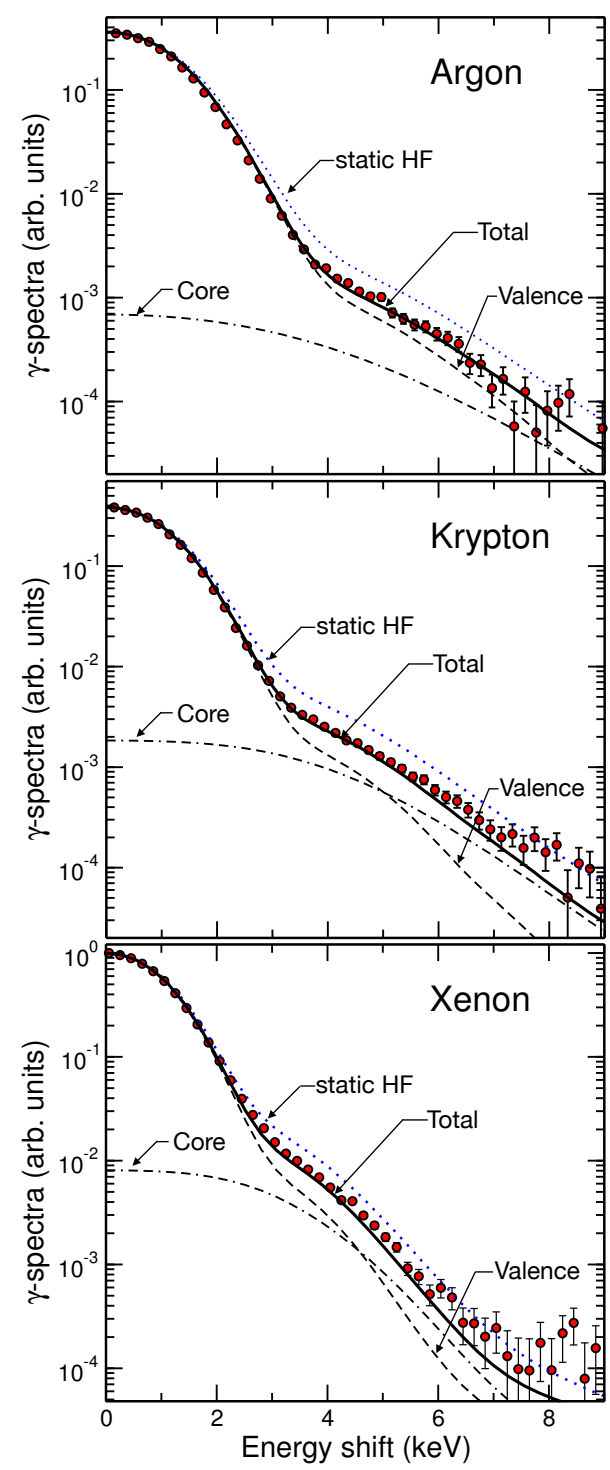

FIG. 4. $\gamma$-spectra for positron annihilation in $\mathrm{Ar}, \mathrm{Kr}$ and Xe. Experiment: red circles. Theory: valence (dashed line); core (dash-dash-dotted line); and total (solid line), calculated with the full annihilation vertex and Dyson positron wave function. Dotted line is the static calculation of [3] (blue dots).

experiment [35]. The corresponding fraction of core annihilation obtained in our MBT calculation is $0.55 \%$ in Ar, $1.53 \%$ in $\mathrm{Kr}$, and $2.23 \%$ in $\mathrm{Xe}$ [36].

The IPA $\gamma$-spectra obtained for the positron in the static atomic field (dotted lines in Fig. 4) are significantly broader than the experiment. Such calculation also overestimates the fraction of core annihilation by a factor of two. However, when this fraction is used as a free parameter to fit the experimental data [3], the core annihilation fractions for $\mathrm{Kr}$ and $\mathrm{Xe}(1.3 \%$ and $2.4 \%$, respectively) are close to the above ab initio values.

Enhancement factors.--In the MBT approach, the en- 


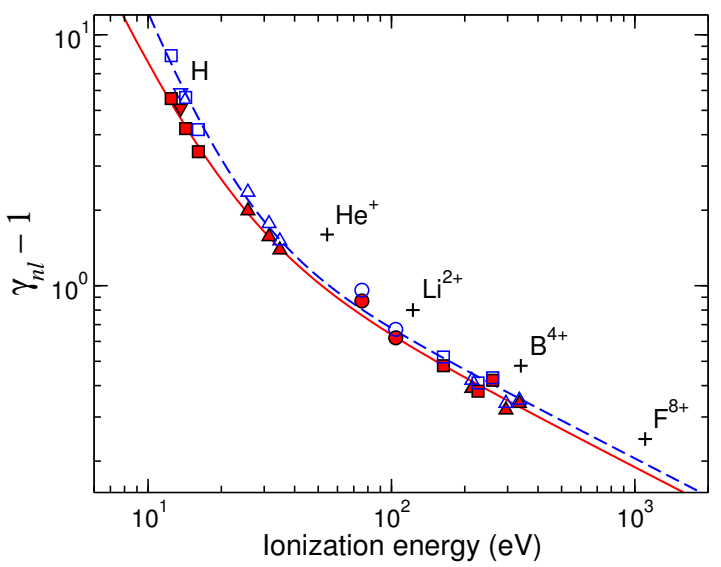

FIG. 5. Enhancement factors (2) calculated using static HF (open symbols) and Dyson (solid symbols) positron states, for the $s$ (triangles), $p$ (squares) and $d$ (circles) valence and core orbitals in $\mathrm{Ar}, \mathrm{Kr}$, and $\mathrm{Xe}$; $1 s$ orbitals hydrogen (upsidedown triangles)[21]; and hydrogen-like ions (plus signs) [19]. Dashed line is the fit (3) of $\gamma_{n l}$ for atoms obtained using the static $\mathrm{HF}$ positron wave function $(A=42.0 \mathrm{eV}, B=24.9 \mathrm{eV}$, $\beta=2.54)$, and the solid line is that for the Dyson positron wave function ( $A=35.7 \mathrm{eV}, B=22.7 \mathrm{eV}, \beta=2.15)$.

hancement factors due to the correlation corrections to the annihilation vertex (Fig. 1), are found from the ratio of the annihilation rate obtained with the full vertex to that of the zeroth-order (IPA), for each electron orbital $n l$ :

$$
\gamma_{n l}=\frac{Z_{\mathrm{eff}, n l}^{(0+1+\Gamma)}}{Z_{\mathrm{eff}, n l}^{(0)}} .
$$

Figure 5 shows the EF $\gamma_{n l}$ for the core and valence orbitals in $\mathrm{Ar}, \mathrm{Kr}$ and Xe, for both static HF and Dyson incident positron states. Also shown are values of $\gamma_{1 s}$ for hydrogen and hydrogen-like ions, from the MBT calculations $[19,21]$.

The values of $\gamma_{n l}$ obtained with the positron wave function in the static atomic field are slightly larger that those found using the fully correlated Dyson wave functions (although this effect is negligible for the positive ions). This difference aside, Fig. 5 displays a near-universal scaling of the EF for the neutral atoms with the orbital ionization energy $I_{n l}$. This scaling can be parametrized by the formula

$$
\gamma_{n l}=1+\sqrt{A / I_{n l}}+\left(B / I_{n l}\right)^{\beta},
$$

where $A, B$ and $\beta$ are constants found by fitting the numerical data. The second term on the right-hand side of (3) describes the effect of the first-order correction, Fig. 1 (b). Its scaling with $I_{n l}$ is motivated by the $1 / Z$ scaling of the EF in hydrogen-like ions [19]. The third term is phenomenological; it accounts for the higherorder corrections which are important for the valence electrons (cf. Fig. 2).
Summary.-Many-body theory has been used to calculate the contribution of individual subshells to the $\gamma$ spectra of positron annihilation in noble gases. Inclusion of core annihilation gives excellent agreement with experiment and yields accurate core annihilation probabilities. The calculated "exact" vertex enhancement factors are found to follow a simple scaling with the electron ionization energy. This result can be used to improve simple IPA calculations of core annihilation on atoms across the periodic table and in condensed matter.

We thank C. M. Surko for valuable discussions. DGG is grateful to H. R. Sadeghpour for hospitality during his visit to ITAMP.

* Correspondences to: dermot.green@balliol.oxon.org; Present address: Joint Quantum Centre (JQC) Durham/ Newcastle, Department of Chemistry, Durham University, South Road, Durham, DH1 3LE, UK.

† g.gribakin@qub.ac.uk

[1] Core electrons, likewise, tunnel through the atomic binding potential towards the positron, and their wave functions are affected by "exchange-assisted tunneling" [37].

[2] K. G. Lynn, J. R. MacDonald, R. A. Boie, L. C. Feldman, J. D. Gabbe, M. F. Robbins, E. Bonderup, and J. Golovchenko, Phys. Rev. Lett. 38, 241 (1977).

[3] K. Iwata, G. F. Gribakin, R. G. Greaves, and C. M. Surko, Phys. Rev. Lett. 79, 39 (1997).

[4] P. Asoka-Kumar, M. Alatalo, V. Ghosh, A. Kruseman, B. Nielsen, and K. Lynn, Phys. Rev. Lett. 77, 2097 (1996).

[5] K. Lynn, J. Dickman, W. Brown, M. Robbins, and E. Bonderup, Phys. Rev. B 20, 3566 (1979).

[6] M. Alatalo, B. Barbiellini, M. Hakala, H. Kauppinen, T. Korhonen, M. Puska, K. Saarinen, P. Hautojärvi, and R. Nieminen, Phys. Rev. B 54, 2397 (1996).

[7] F. Tuomisto and I. Makkonen, Rev. Mod. Phys. 85, 1583 (2013).

[8] A. Weiss, R. Mayer, M. Jibaly, C. Lei, D. Mehl, and K. G. Lynn, Phys. Rev. Lett. 61, 2245 (1988).

[9] T. Ohdaira, R. Suzuki, T. Mikado, H. Ohgaki, M. Chiwaki, and T. Yamazaki, Appl. Surf. Sci. 116, 177 (1997).

[10] A. H. Weiss, N. G. Fazleev, M. P. Nadesalingam, S. Mukherjee, S. Xie, J. Zhu, and B. R. Davis, Radiat. Phys. Chem. 76, 285 (2007).

[11] J. Mayer, C. Hugenschmidt, and K. Schreckenbach, Surface Science 604, 1772 (2010).

[12] C. Hugenschmidt, B. Lwe, J. Mayer, C. Piochacz, P. Pikart, R. Repper, M. Stadlbauer, and K. Schreckenbach, Nuc. Instrum. Meth. A 593, 616 (2008).

[13] J. Mayer, C. Hugenschmidt, and K. Schreckenbach, Phys. Rev. Lett. 105, 207401 (2010).

[14] A. Eshed, S. Goktepeli, A. Koymen, S. Kim, W. Chen, D. O'Kelly, P. Sterne, and A. Weiss, Phys. Rev. Lett. 89, 075503 (2002).

[15] S. Kim, A. Eshed, S. Goktepeli, P. Sterne, A. Koymen, W. Chen, and A. Weiss, Phys. Rev. B 73, 014114 (2006).

[16] K. O. Jensen and A. Weiss, Phys. Rev. B 41, 3928 (1990).

[17] D. G. Green, J. A. Ludlow, and G. F. Gribakin, Phys. Rev. A 90, 032712 (2014). 
[18] L. J. M. Dunlop and G. F. Gribakin, J. Phys. B 39, 1647 (2006).

[19] D. G. Green and G. F. Gribakin, Phys. Rev. A 88, 032708 (2013).

[20] D. G. Green, S. Saha, F. Wang, G. F. Gribakin, and C. M. Surko, New J. Phys. 14, 035021 (2012).

[21] G. F. Gribakin and J. Ludlow, Phys. Rev. A 70, 032720 (2004).

[22] S. Kahana, Phys. Rev. 129, 1622 (1963).

[23] J. P. Carbotte, Phys. Rev. 155, 197 (1967).

[24] E. Boroński and R. Nieminen, Phys. Rev. B 34, 3820 (1986).

[25] M. J. Puska and R. M. Nieminen, Rev. Mod. Phys. 66, 841 (1994).

[26] J. Arponen and E. Pajanne, Annals of Physics 121, 343 (1979).

[27] E. Bonderup, J. U. Andersen, and D. N. Lowy, Phys. Rev. B 20, 883 (1979).

[28] J. Mitroy and B. Barbiellini, Phys. Rev. B 65, 235103 (2002).

[29] The theory also allows one to extract momentumdependent EF [19, 20].

[30] V. B. Berestetskii, E. M. Lifshitz, and L. P. Pitaevskii, Quantum Electrodynamics, 2nd ed. (Pergamon, Oxford,
1982).

[31] D. G. Green and G. F. Gribakin, (unpublished).

[32] D. G. Green, Ph.D. thesis, Queen's University Belfast (2011)

[33] $Z_{\text {eff }}$ is the ratio of the positron annihilation rate in an atomic or molecular gas to the Dirac annihilation rate in the electron gas of the same number density.

[34] All calculations are done for the $s$-wave incident positron with room-temperature momentum $k=0.04$ a.u.

[35] In Kr and Xe the theoretical spectrum slightly underestimates the measurements at large $\epsilon$. This could be due to the neglect of relativistic effects on the electron wave functions [38]. However, a recent work [39] which employs model potentials to describe positron-atom interactions, shows that the relativistic effect on the $\gamma$-spectra is small, e.g., increasing the FWHM in Xe by only $1.4 \%$.

[36] The uncertainty of these numbers is about $5 \%$, comparable to effect of nonladder 3rd-order diagrams on the valence annihilation rates [17].

[37] M. G. Kozlov and V. V. Flambaum, Phys. Rev. A 87, 042511 (2013).

[38] J. P. D. Cook, J. Mitroy, and E. Weigold, Phys. Rev. Lett. 52, 1116 (1984).

[39] Y. Cheng and J. Mitroy, Phys. Rev. A 90, 042702 (2014). 